\title{
Frank-van der Merwe Growth in Bilayer Graphene
}

Haozhe Wang ${ }^{1 \dagger}$, Zhenpeng $\mathrm{Yao}^{2, \dagger}$, Gang Seob Jung ${ }^{3}$, Qichen Song ${ }^{4}$, Marek Hempel ${ }^{1}$, Tomas Palacios ${ }^{1}$, Gang Chen ${ }^{4}$, Markus J. Buehler ${ }^{3}$, Alán Aspuru-Guzik ${ }^{2,5,6,7}$ and Jing Kong ${ }^{1 *}$

${ }^{I}$ Department of Electrical Engineering and Computer Science, Massachusetts Institute of Technology, Cambridge, MA 02139, USA

${ }^{2}$ Department of Chemistry and Chemical Biology, Harvard University, Cambridge, MA 02138, USA

${ }^{3}$ Department of Civil and Environment Engineering, Massachusetts Institute of Technology, Cambridge, MA 02139, USA

${ }^{4}$ Department of Mechanical Engineering, Massachusetts Institute of Technology, Cambridge, MA 02139, USA

${ }^{5}$ Department of Chemistry and Department of Computer Science, University of Toronto, Toronto, Ontario M5S 3H6, Canada

${ }^{6}$ Vector Institute for Artificial Intelligence, Toronto, Ontario M5S 1M1, Canada

${ }^{7}$ Canadian Institute for Advanced Research (CIFAR) Senior Fellow, Toronto, Ontario M5S 1M1, Canada

*Corresponding author. Email: jingkong@mit.edu (J.K.)

†These authors contributed equally to this work.

\begin{abstract}
Bilayer graphene has attracted significant interests due to its unique properties, including fascinating electrical behavior when one layer is slightly rotated relative to the other. However, the quality of large-area bilayer graphene is often limited by the layer-plus-island growth mode in which islands of thicker graphene present as unavoidable impurities. Here, we report the observation of the layer-by-layer, Frank-van der Merwe (FM) growth mode in bilayer graphene where multilayer impurities are suppressed. Instead of the conventional surface adhesive energy, it is found that interface adhesive energy is possible to be tuned with an oxidative pretreatment. The FM-grown bilayer graphene is of AB-stacking or with small-twisting-angle $\left(\theta=0-5^{\circ}\right)$, which is more mechanically robust compared to monolayer graphene, facilitating a free-standing wet transfer technology.
\end{abstract}


High-quality synthesis, systematic characterization and clean transfer of large-area bilayer graphene (2LG) are important for transforming the unusual physical properties of 2LG into disruptive technologies [1-3]. Specifically, small-twisting-angle 2LG $\left(\theta=0-5^{\circ}\right)$ exhibits fascinating properties, including superconductivity [4-7] and electrically tunable bandgaps [8-10] compared to other 2LG with larger twisting angles [11]. To further transfer these physical observations into disruptive innovations, many efforts have been made to target large-scale highquality 2LG synthesis using chemical vapor deposition (CVD)[12-14]. Significant progress has been made with CVD 2LG synthesis [15-18], particularly, wafer-scale growth of uniform AB-stacked bilayer graphene film reported recently on liquid $\mathrm{Pt}_{3} \mathrm{Si} /$ solid $\mathrm{Pt}$ substrate[15] and $\mathrm{AB}$-stacked bilayer or multilayer graphene larger than a centimeter using $\mathrm{Cu} / \mathrm{Ni}(111)$ substrate[18]. Some of these methods are via controlling the cooling to let the dissolved carbon to precipitate out[15-18], or others through "pocket" approach [19]. Particularly, in the pocket method the most common observation is that the synthesis follow a Stranski-Krastanov (SK) mode [20, 21], in which growth within a layer and nucleation of additional layers unavoidably occur at the same time. This method makes it unlikely that only $2 \mathrm{LG}$ forms uniformly over a large area as regions of single layer graphene (1LG) or other multilayers (3LG, 4LG, etc.) always exist.

We report uniform growth of 2LG using the Frank-van der Merwe (FM) growth mode in CVD graphene [20] using the pocket approach. In the FM mode, the existing layer continues to grow and merge into a film prior to the nucleation of an additional layer. This requires the energy to nucleate a new layer (i.e., surface adhesive energy) is much larger than the energy to grow the existing layer (i.e., atomic cohesive energy). Previous studies found that the growth of additional graphene layers happens at the interface of the existing graphene layer and the $\mathrm{Cu}$ substrate instead of growing on top of the existing layer [19, 22]. By tuning the interface adhesive energy, our calculations and experiments both show that the $3^{\text {rd }} \mathrm{LG}$ nucleation can be substantially suppressed to favor the $2^{\text {nd }} \mathrm{LG}$ growth. Although a fully continuous 2LG film is not achieved in this study yet, the occurrence of graphene multilayers is substantially suppressed in the FM-mode-grown samples compared to that of SK mode.

Through first-principle calculations, the likelihood of an incoming carbon atom for $3^{\text {rd }} \mathrm{LG}$ nucleation versus to extend the $2^{\text {nd }} L G$ growth at the interface of first layer of graphene $\left(1^{\text {st }} L G\right)$ and modified Cu were estimated (Fig. 1A). In the $\mathrm{Cu}$ growth substrate, we chose oxygen atoms as adatoms, because they promote graphene growth with larger crystal sizes [23] and facilitate the dehydrogenation of the carbon precursor leading to large single-crystal bilayer graphene growth as well [19]. For comparison, we constructed a model with the same configuration, except that the $\mathrm{Cu}$ substrate is original and contains no adatoms (Fig. 1B) and determined the carbon atom formation energies at eight different locations (Fig. 1C). The atomic cohesive energies of $2^{\text {nd }}$ LG are typically much smaller compared to the interface adhesive energies of $3^{\text {rd }}$ LG nucleation. This relative difference implies that an incoming carbon atom should extend the $2^{\text {nd }} \mathrm{LG}$ growth, rather than nucleating the $3^{\text {rd }} \mathrm{LG}$ for both bare and modified $\mathrm{Cu}$ substrates. Compared to bare $\mathrm{Cu}$, the interface adhesive energy required for $3^{\text {rd }} \mathrm{LG}$ nucleation on the modified $\mathrm{Cu}$ substrate $\left(\mathrm{Cu}_{\mathrm{x}} \mathrm{O}_{1-\mathrm{x}}\right)$ is much higher (Fig. 1C, 1D). The $\Delta \mathrm{E}$ of $\mathrm{Cu}_{\mathrm{x}} \mathrm{O}_{1-\mathrm{x}}$ is twice that of the bare $\mathrm{Cu}(1.82 \mathrm{eV} / \mathrm{C}$ versus $3.73 \mathrm{eV} / \mathrm{C}$ ), indicating that the incorporation of oxygen atoms on $\mathrm{Cu}$ surface can substantially suppress the $3^{\text {rd }} \mathrm{LG}$ nucleation. We can calculate the Boltzmann factor, which is the ratio of probabilities of two events $\left(2^{\text {nd }} \mathrm{LG}\right.$ growth versus $3^{\text {rd }}$ LG nucleation), using: 


$$
\frac{\exp \left(-\frac{\Delta E_{F 2}+\Delta E_{M 2}}{k_{\mathrm{B}} \mathrm{T}}\right)}{\exp \left(-\frac{\Delta E_{F 3}+\Delta E_{M 3}}{k_{\mathrm{B}} \mathrm{T}}\right)}=\exp \left(-\frac{\Delta E_{F 2}-\Delta E_{F 3}}{k_{\mathrm{B}} \mathrm{T}}\right) \exp \left(-\frac{\Delta E_{M 2}-\Delta E_{M 3}}{k_{\mathrm{B}} \mathrm{T}}\right)
$$

where $\Delta E_{F i}$ (Fig. 1C) and $\Delta E_{M i}$ are the formation energy and migration barrier of an incoming carbon atom attaches to the $i^{\text {th }}$ layer of graphene, respectively, $k_{B}$ is the Boltzmann constant, and $T$ is the growth temperature (i.e. 1050 ${ }^{\circ} \mathrm{C}$ ). Assuming the $3^{\text {rd }} \mathrm{LG}$ nucleation requires no further interface diffusion, $\Delta E_{M 3} \sim 0$, and the reported activation energy for a $\mathrm{C}$ atom to perform both bulk and interface diffusion, $\Delta E_{M 2}$ is $1.42 \mathrm{eV}$ [10], the Boltzmann factor of $2^{\text {nd }} \mathrm{LG}$ growth versus $3^{\text {rd }} \mathrm{LG}$ nucleation on pure and modified $\mathrm{Cu}$ substrates are $3.2 \times 10^{1}$ and $7.1 \times 10^{8}$, respectively. This implies that if we have a $2 \mathrm{LG}$ film of $1 \mathrm{~cm}^{2}$ size, the maximum area that the $3^{\text {rd }}$ LG can grow at the $2^{\text {nd }}$ LG/substrate interface for both $\mathrm{Cu}$ and $\mathrm{Cu}_{\mathrm{x}} \mathrm{O}_{1-\mathrm{x}}$ are $3.1 \mathrm{~mm}^{2}$ and $0.14 \mu \mathrm{m}^{2}$, respectively (See Supplemental Materials). This suggests that centimeter-scale, high-purity $2 \mathrm{LG}$ film can be realized by tuning the interface adhesive energy.

We implemented this strategy by using a mild oxidation pretreatment step integrated into the CVD "pocket" approach (Fig. 1E) ( See Supplemental Materials). For comparison, the same growth steps are carried out for both bare and treated $\mathrm{Cu}$ foil. For graphene samples synthesized on bare $\mathrm{Cu}$, we observed random growth of $3 \mathrm{LG}$ islands and inevitable nucleation of thicker layers, similar to previous reports $[22,24]$. For graphene samples synthesized on treated $\mathrm{Cu}$, we observed no 3LG impurities (Fig. 1E). We only saw $1 \mathrm{LG}$ and $2 \mathrm{LG}$ regions under an optical microscope over an area of $2 \mathrm{~cm}^{2}$. We performed the same comparison studies on two types of commercial $\mathrm{Cu}$ foils (Alfa Aesar 13380 and 46986; Fig. 1F) and observed successful suppression of the $3^{\text {rd }}$ LG nucleation, indicating that this mechanism is not limited to the specific types of $\mathrm{Cu}$ foils. In addition, we conducted a series of surface analyses on the treated $\mathrm{Cu}$ and found that our pre-treatment modifies the surface stoichiometry of a bare $\mathrm{Cu}$ to $\mathrm{Cu}_{1.7} \mathrm{O}$. This surface-level oxidation enhances as we increase the pre-treatment duration (Fig. S1). It is notable that XPS spectra only show $\mathrm{Cu}_{2} \mathrm{O}$ features but no $\mathrm{Cu}^{2+}(\mathrm{CuO})$ peaks, therefore the oxidized phase should be $\mathrm{Cu}_{2} \mathrm{O}$.

In the current study, galaxy-like 2LG morphologies were obtained at the centimeter-scale ${ }^{\text {st }} \mathrm{LG} / \mathrm{Cu}$ interface.. Over a 1 x $1 \mathrm{~mm}^{2}$ optical imaging area, we observed merging of 2LG domains into an elliptical film surrounded by numerous 2LG sparse domains and 1LG regions (Fig. 1H). Similar graphene ad-layer morphologies were observed regardless of whether the $\mathrm{Cu}$ was treated (Fig. S2). We attributed this galaxy-like growth morphology to the sparse locations of bulk diffusion of carbon atoms (i.e. from $\mathrm{Cu}$ interior to exterior through the foil thickness), and the limited interface-diffusion length of carbon atoms at the $1^{\text {st }} \mathrm{LG} / \mathrm{Cu}$ interface [25], corresponding to Steps (ii) and (iii) in Fig. 1G (See Supplemental Materials). Bulk diffusion may happen via preferred paths, due to the presence of uncontrollable impurities in the commercial $\mathrm{Cu}$ foil [26]. Carbon atoms that penetrated through bulk $\mathrm{Cu}$ will emerge at several preferred points within the $\mathrm{Cu}$ surface lattice and start to migrate via interface diffusion. According to diffusion theories, carbon concentration surrounding a penetration point follows Gaussian distributions [27]. Engineering of the treated $\mathrm{Cu}$ to provide increased bulk diffusion paths should increase the area coverage of $2 \mathrm{LG}$.

We statistically analyzed the SK and FM-mode-grown samples using optical microscopy (Fig. 2A, C). On the bare $\mathrm{Cu}$ substrate, the SK growth mode appears to be dominant (Fig. 2A, 2B). We found 2LG domains using the 
SK growth mode with an average size of $\sim 10 \mu \mathrm{m}$, but multi-layer impurities appear even when the growth is limited to one hour. As the growth duration increased from $1 \mathrm{~h}$ to $4 \mathrm{~h}$, bilayer graphene domains get larger in size and eventually merge into a film. However, these are accompanied by not only the concurrent growth of 3LG and 4LG impurities, but also the nucleation of subsequent layers up to 8LG. In contrast, when we used treated $\mathrm{Cu}$ for one hour, most of the $2 \mathrm{LG}$ domains synthesized on the treated $\mathrm{Cu}$ substrate of regular hexagonal shape (Fig. 2C). The domain size of the $2 \mathrm{LG}$ increased with growth duration, and we observed no $3^{\text {rd }} \mathrm{LG}$ nucleation for up to three hours (Fig. 2C, D). Our typical 3.33 h-grown sample had connection of 2LG domains into a continuous area. We observe the appearance of a $\mu \mathrm{m}$-sized $3 \mathrm{LG}$ domain in a continuous $2 \mathrm{LG}$ region for our typical 4 h-grown samples, but without any 4LG impurities (Fig. 2C).

To quantitively evaluate the suppression of multilayer impurities in our FM-mode-grown bilayer graphene film, we developed a statistical method with the help of microscopy analysis. Specifically, we first converted the original microscopy images of graphene films transferred on $\mathrm{Si} / \mathrm{SiO}_{2}$ substrates into grayscale and plotted the corresponding pixel counts (5,002,624 pixels for each image). A histogram for a typical FM-mode grown graphene grown for one hour contains only two peaks (1LG and 2LG), while that of SK-mode contains four peaks, which can be attributed to $1 \mathrm{LG}, 2 \mathrm{LG}, 3 \mathrm{LG}$ and 4LG (Fig. 2E). To systematically label graphene region with different layer numbers, we assigned a range of gray levels to each histogram peak and converted the corresponding pixels into red (Fig. 2E). Upon layer labelling in each microscopy image, we estimated the occurrence probability of 2LG and multilayer impurities by extracting the ratio of pixel counts. For this analysis (Fig. 2F), all samples were grown for $3.33 \mathrm{~h}$. We randomly acquired a 10 microscopy images, with sizes of at most $40 \times 40 \mu \mathrm{m}^{2}$, from both the SKand the FM-mode grown graphene samples (Fig. 2F). The occurrence probability of 2LG is less than half $(46.20 \pm$ $14.22 \%$ ) for the SK-mode grown samples, while that of FM-mode is close to $100 \%$ (99.32 $\pm 0.34 \%)$.

We found using Raman spectroscopy that our FM-mode grown bilayer graphene are quasi-AB stacked (i.e., either AB-stacked or having a small twisting angle $\left.\left(\theta=0-5^{\circ}\right)\right)$ (Fig. 3A) [28, 29]. From our transmittance electron microscopy (TEM) selected area electron diffraction (SAED) characterization of multiple locations in our FM-2LG sample, AB stacking have been observed (Fig. S3); nevertheless, since SAED only examines limited sample areas, one cannot conclude that all 2LG regions are AB-stacked. Rather, Raman spectroscopy mapping gives us characterization of large area regions, and with 2D band's full width at half maximum (2D FWHM) one can narrow down the twisting angle $\theta$ to be $<5^{\circ}$ [29]. A typical 1LG Raman signature is shown in Fig 3A, spot 1 with the positions of $G$ and $2 D$ bands $\left(\omega_{G}\right.$ and $\left.\omega_{2 D}\right)$ located at 1582 and $2670 \mathrm{~cm}^{-1}$, respectively. The intensity ratio of 2D to $\mathrm{G}$ bands $\left(\mathrm{I}_{2 \mathrm{D}} / \mathrm{I}_{\mathrm{G}}\right)$ is $\sim 2$, and the $2 \mathrm{D}$ full width at half maximum $\left(\Gamma_{2 D}\right)$ is $34 \mathrm{~cm}^{-1}$. The other spots exhibited an almost identical Raman spectra with $\omega_{G}=1580 \mathrm{~cm}^{-1}, \omega_{2 D}=2680 \mathrm{~cm}^{-1}, \mathrm{I}_{2 \mathrm{D}} / \mathrm{I}_{\mathrm{G}}=\sim 1$, and $\Gamma_{2 D}=58 \mathrm{~cm}^{-1}$. These features all can be identified as 2LG with either AB-stacked or small twisting angles $\left(\theta=0-5^{\circ}\right)[28,29]$. We obtained consistent results from 60 different Raman spectra, that we randomly collected from 6 different samples, confirming our growth method produces quasi-AB stacked 2LG. In contrast, for the SK-mode grown graphene sample (Fig. 3B), the Raman spectra taken at 4 typical spots all had different $\omega_{G}, \omega_{2 D}, \mathrm{I}_{2 \mathrm{D}} / \mathrm{I}_{\mathrm{G}}$, and $\Gamma_{2 D}$. We identified the Raman spectra of spots 1 and 4 in Fig. 3B 1LG and 3LG, respectively. Under the optical microscope, we recognized spots 2 and 3 as 2 LG because they shared the same optical contrast, which is at the interval of 1LG and 
3LG. One spot was $2 \mathrm{LG}$ with a small-twisting-angle $\left(\mathrm{I}_{2 \mathrm{D}} / \mathrm{I}_{\mathrm{G}}=0.77\right.$ and $\left.\Gamma_{2 D}=62 \mathrm{~cm}^{-1}\right)$ and the other had a largertwisting-angle $2 \mathrm{LG}\left(\mathrm{I}_{2 \mathrm{D}} / \mathrm{I}_{\mathrm{G}}=2.3\right.$ and $\left.\Gamma_{2 D}=37 \mathrm{~cm}^{-1}\right)$ [29]. The variation of twisting angles in the SK-mode-grown 2LG is large, unlike those in the FM-mode.

We also performed Raman mappings on both FM- and SK-mode-grown graphene samples that we transferred onto $\mathrm{Si} / \mathrm{SiO}_{2}$. For the FM-mode sample, we found $\Gamma_{2 D}$ the remains constant throughout the single domain or two merging domains of 2LG (Fig. 3C). We collected Raman spectra from typical SK-mode samples, over an area that consists of 1LG, 2LG, 3LG, and multilayer graphene (MLG) (Fig. 3D). Surprisingly, we classified the 2LG region, which looks uniform under optical microscope, into at least 2 divisions in the Raman map of $\Gamma_{2 D}$ (Fig. 3D). We further classified these Raman mapping data using a simple and fast unsupervised learning approach, the k-means algorithm, which measures dissimilarity between two data points using square Euclidean distance (See Supplemental Materials). We considered all 5 parameters $\left(\omega_{G}, \omega_{2 D}, \mathrm{I}_{2 \mathrm{D}}, \mathrm{I}_{\mathrm{G}}\right.$, and $\left.\Gamma_{2 D}\right)$ in our artificial-intelligenceassisted Raman data classification. To visualize the classification results, we selected 3 parameters $\left(\omega_{G}, \omega_{2 D}\right.$, and $\Gamma_{2 D}$ ) and made three-dimensional plots (Fig. 4A, C). The algorithm clustered all 18,000 Raman spectra into two classes in several seconds. By reading the mean value $\left(\overline{\omega_{G}}, \overline{\omega_{2 D}}, \overline{\Gamma_{2 D}}\right)$ of each cluster, we identified Class I and II as the Raman data of 1LG and quasi-AB-stacked 2LG, respectively (Fig. 4B). Error rate of our AI-assisted Raman analysis is 0.027 , as cross-verified by hierarchical clustering algorithm. We also verified the quasi-AB-stacked $2 \mathrm{LG}$ (Class II) using TEM (Fig. S3). We executed the same algorithm for comparison on the Raman data of SK-mode sample (Fig. 4C), where Class I to V in Fig. 4D represents 1LG, quasi-AB-stacked 2LG, larger-twisting-angle 2LG, 3LG, and MLG, respectively. Using the artificial-intelligence-assigned class number (I to V), we constructed a spatial map (Fig. S4), where the boundaries/edges that separate $1 \mathrm{LG}, 2 \mathrm{LG}$, and 3LG etc. can be recognized. For graphene samples on substrates without a good optical contrast such as glass, quartz or sapphire, the Raman mapping or this facile classification method will be very useful to identify regions of different layer numbers and stacking orders.

Utilizing the mechanical advantage of 2LG in comparison to $1 \mathrm{LG}$ [30], we devised a support-free wet transfer approach driven by Marangoni effect. Owing to graphene's thinness, a transfer support layer is generally required to prevent cracks' generation and propagation. In the conventional polymer-supported CVD graphene transfers [31, 32], a polymer sacrificial support layer was first coated on a piece of graphene/Cu. The coated sample was then floated on an etchant solution to completely remove the $\mathrm{Cu}$. The sample was then rinsed with deionized water for at least three times. For these rinsing steps, a flat scoop (e.g., Si wafer or glass slide) was used to move the sample from one beaker of deionized water to another, and an external force (i.e., tweezers or glass slide) was required to drive the sample from liquid surface to the flat scoop, until the sample is free of $\mathrm{Cu}$ etchant residues. If these rinsing steps were carried out without the polymer support layer, the external force usually generates cracks and tears in the graphene layer. To eliminate the need of sacrificial transfer support layer, we substitute the external force with Marangoni-driven internal forces, which are the hydrodynamic forces generated whenever liquids with different surface tensions mix [33]. To achieve these, prior to sample loading, we fine-tuned the liquids' surface tension with the help of isopropyl alcohol (IPA). We first wetted a flat scoop with a liquid of higher surface tension (high- $\sigma$ ), and quickly dipped it into the lower-surface-tension liquid with a floating graphene sample. Forced by Marangoni 
flows, the sample will automatically climb from liquid surface to the flat scoop, in a few seconds (Movie S1). When we gently dipped the flat scoop back into the high- $\boldsymbol{\sigma}$ liquid, the graphene sample gradually glides to the liquid surface (See Supplemental Materials). Fig. 5D shows a typical image of a centimeter-scale 2LG sample transferred on $\mathrm{Si} / \mathrm{SiO}_{2}$ substrate, using our free-standing Marangoni-driven graphene transfer process. We found the yield (in terms of area percentage for un-broken regions) of this transfer approach for our 2LG is $>95 \%$, whereas for $1 \mathrm{LG}$ is $\sim 66.7 \%$ (Fig. S5; Movie S2). This observation was consistent with our molecular dynamic simulations, ABstacked 2LG was found to be robust under the Marangoni flow, even in the presence of atomic defects (Fig. 5B and C; Movie S3). A frame made by Scotch tape or silver paste was made on the surface of graphene outlining the four edges for bootstrapping the area in our method, similar to the marker frame transfer approach [34], though the Marangoni effect simplify the transfer process and avoid using a dedicated experimental setup to exchange the etching/rinsing solution.. We anticipate this polymer-free, Marangoni-flow-driven transfer approach developed on our FM-mode-grown 2LG can be generalized to other few-layer 2D materials.

\section{Methods}

Cu substrate pre-treatment and bilayer graphene growth: $\mathrm{Cu}$ foil purchased from Alfa Aesar (\#46365) with 25 $\mu \mathrm{m}$ thickness and $99.8 \%$ purity was used as the growth substrate in this work. Prior to growth, the $\mathrm{Cu}$ substrate was first subjected to a Ni etchant (Nickel Etchant TFB, Transense) to remove surface contaminations. To enable Frankvan der Merwe (FM) growth mode, the substrate was then annealed in ambient conditions at $130{ }^{\circ} \mathrm{C}$ for $3 \mathrm{~h}$. We note that this step was omitted for all SK-mode grown samples. After substrate pre-treatment, the $\mathrm{Cu}$ foils were folded into a pocket, as discussed in previous work[19], and loaded into a low-pressure chemical vapor deposition (LPCVD) system. Temperature of the LPCVD system was then ramped up $1050{ }^{\circ} \mathrm{C}$ in $30 \mathrm{~min}$ (50 mTorr). In the next $30 \mathrm{~min}$, the LPCVD system was maintained at $1050^{\circ} \mathrm{C}$. Following which, $50 \mathrm{sccm}$ of $\mathrm{H}_{2}$ gas was introduced to the system, and $1 \mathrm{~min}$ later, $1 \mathrm{sccm}$ of $\mathrm{CH}_{4}$ was added to the system to activate the growth of $2 \mathrm{LG}$. The system was maintained at the same conditions until a desired growth duration ends (e.g., $3.33 \mathrm{~h}$ ). We note all graphene in this work were grown using $\mathrm{Cu}$ foil purchased from Alfa Aesar (\#46365), except for those shown Fig. 1F and S4. For Alfa Aesar Cu foil (\#13380) with $127 \mu \mathrm{m}$ thickness and 99.9\% purity, we observe small arbitrary shapes bilayer graphene growth and successful suppression of additional graphene layers (Fig. 1F). For Alfa Aesar Cu foil (\#46986) with $25 \mu \mathrm{m}$ thickness and 99.8\% purity (labeled as 'coated'), we observe no evidence of bilayer graphene growth (Fig. S6) .

PMMA-assisted graphene transfer: Notably, 2LG is synthesized at the exterior side of a $\mathrm{Cu}$ pocket, using the LPCVD approach described above. To transfer the 2LG from growth substrate to a destination substrate, the Cu pocket was carefully cut, with extra care given to the exterior side of $\mathrm{Cu}$ pocket. All samples characterized in this work were transferred on $\mathrm{Si} / \mathrm{SiO}_{2}$ substrates with the help of polymethylmethacrylate (PMMA) support layers, unless otherwise specified. In the PMMA-supported graphene transfer, a $300 \mathrm{~nm}$-thick layer of PMMA 950 A5 (Microchem Inc.) was first spun-coated on the graphene/Cu sample followed by a one hour of $80^{\circ} \mathrm{C}$ oven baking. 
To remove the $\mathrm{Cu}$ growth substrate, the sample was then floated on top of $\mathrm{Cu}$ etchant (Copper Etchant TFB, Transense) for half an hour. Following which, the sample was rinsed with deionized water for at least thrice. For these steps of sample rinsing, a flat scoop (i.e., Si wafer or glass slide) is used to ship the sample from one beaker of deionized water to another, and an external force (i.e., tweezers) is required to drive the sample from liquid surface to the flat scoop, until the sample is free of $\mathrm{Cu}$ etchant residues. Finally, the destination substrate (i.e., $\mathrm{Si} / \mathrm{SiO}_{2}$ substrate) was used to fish the graphene film. To expedite sample drying, the sample was initially blew-dry with nitrogen flow, followed by an $8 \mathrm{~h}$ of $80^{\circ} \mathrm{C}$ oven baking. To remove the sacrificial PMMA support layer, the sample must be then soaked in acetone for $6 \mathrm{~h}$, rinsed with IPA, and finally blew dry with nitrogen.

Support-free Marangoni-driven graphene transfer: Similar to PMMA-assisted transfer, $\mathrm{Cu}$ pocket was first opened to obtain the 2LG grown at the exterior side. Silver paint (PELCO. Inc) or Scotch tape was then used to make a frame surrounding the region of interest, such that the operator can locate borders of the diced sample. Subsequently, the support-free 2LG sample was floated on top of the $\mathrm{Cu}$ etchant for 30 min to etch off the $\mathrm{Cu}$ substrate. In the subsequent rinsing process, the flat scoop was made more hydrophilic with an oxygen plasma treatment and the surface tension of deionized water was tuned by diluting with different amount of Isopropyl Alcohol (IPA). Specifically, a feasible combination of IPA concentrations is 5\%, 2\%, 1.5\%, 1\%, and $0 \%$. The support-free graphene sample was initially shipped from a solution with the least surface tension to another solution with the second-lowest surface tension, and so on, until the desired rinsing results achieved. At the last rinsing step, a destination substrate was pre-wetted with pure water (highest surface tension) and dipped into the solution with second-largest-surface-tension, which graphene sample is floating on it. Graphene sample will automatically climb onto the destination substrate, as schematically illustrated in Fig. 5A. For sample drying, blow dry is not recommended, and to expedite the process, an $8 \mathrm{~h}$ of $80^{\circ} \mathrm{C}$ oven baking can be performed. After removing frame by a blade, the sample (i.e., $2 \mathrm{LG}$ transferred on $\mathrm{Si} / \mathrm{SiO}_{2}$ substrate) is now ready to use. We also compared the electrical properties of PMMA-transferred 1LG graphene samples with and without Marangoni rinsing (Fig. S14)

Materials Characterization: Field-emission scanning electron microscopy was conducted using a Zeiss Merlin High-resolution SEM. Raman spectroscopy was performed by Horiba LabRam (spot analysis) and WiTec Alfa 300 (mapping analysis). Transmission electron microscopy was performed using FEI Tecnai Multipurpose Digital TEM. The X-Ray Photoelectron Spectroscopy was performed by Physical Electronics Versaprobe II X-ray Photoelectron Spectrometer.

First-Principle Calculations: The calculations were performed using the Vienna Ab-initio Simulation Package (VASP) [35, 36] within the projector augmented wave (PAW) [37] formalism and the Perdew-Becke-Ernzerhof (PBE) [38] approximation to the exchange-correlation potential was employed. We used a plane wave basis with a cutoff energy of $520 \mathrm{eV}$ and gamma-centered k-meshes, with a density of $8000 \mathrm{k}$-points per reciprocal atom. We also adopted the vdW-DF functional (optB88) [39], which employs a self-consistent van der Waals (vdW) correction. To simulate the nucleation and growth of graphene on the copper surface, we built a surface model with a $4 " \times 4 " \mathrm{Cu}(111)$ surface of 6 layers with a vacuum spacing of $15 \AA$ and the bottom layer is fixed in the 
perpendicular direction to the surface (Fig. S7A) following the previous study[19]. To simulate the surface pretreatment, a similar oxidized $\mathrm{Cu}(111)$ surface model was built with $\mathrm{Cu} / \mathrm{O}$ atomic configurations reproducing the counterpart in $\mathrm{Cu}_{2} \mathrm{O}$ (Fig. S7A). Addition of the $\mathrm{O}$ ions to the system led to two geometrically different ways to introduce graphene to the oxidized surface (Fig. S7B). Both configurations were relaxed in density functional theory (DFT) with the one with lower total energy (O locates in the graphene ring center) chosen to be the configuration of the first graphene layer $\left(1^{\text {st }} \mathrm{LG}\right)$. Additions of the $2^{\text {nd }}$ and $3^{\text {rd }} \mathrm{LG}$ proceeded in an A-B stacking manner (Fig. S9).

Artificial Intelligence Data Analysis: We applied $k$-means clustering to separate unlabeled data samples into $k$ groups with square Euclidean distance as a dissimilarity measure between two data points. In this unsupervised learning algorithm, all data points were clustered based on similarity. In another words, dissimilarity was minimized within each cluster. Specifically, dissimilarity between any data point and a cluster center is the distance from the data point to the cluster center. Therefore, the dissimilarity within a cluster will be the sum over the distances between data points in that cluster and the cluster center. Summation of all cluster dissimilarities is called global dissimilarity, which can be calculated by iterating over each cluster, over each data point within the cluster, and over each feature of the data point:

$$
\sum_{k=1}^{K} \sum_{n: X_{n} \in S_{k}} \sum_{d=1}^{D}\left(x_{n, d}-\mu_{k, d}\right)^{2}
$$

where $k$ is the number of clusters, $n$ is the number of data point, $d$ is the number of features, and $\mu_{k, d}$ is the mean of the cluster. Since we do not know the cluster centers of Raman data in advance, we initialize them with a set of values randomly picked from the existing data points. Subsequently, two steps were performed alternately: (i) assign each data point to the cluster with the closest cluster center, and (ii) update the cluster center to be the mean of all the data points in its cluster. Iteration of these two steps continues until a convergence point found. We note that $k$-means function from scikit-learn package was used in this work.

Molecular Dynamics Simulations: The molecular dynamics (MD) simulations in this study were performed using a LAMMPS [40] package. We utilized an Adaptive Intermolecular Reactive Empirical Bond Order (AIREBO) [41, 42] potential to model the interaction between carbon atoms. The potential is an extended version of the Brenner's Reactive Empirical Bond Order (REBO) potential of the hydrocarbon system [41], which only could describe the short-range interaction $(\mathrm{r}<2 \AA)$. Stuart et al. have expanded REBO by including the four bodies torsional term and Lennard-Jones (LJ) potential based van der Waals (vdW) terms [42], which describe layer-to-layer interaction in a bilayer graphene system. Instead of utilizing the original form of AIREBO, the radius cutoff of the switching function of C-C interaction is set to $2 \AA$ for both $R_{\min }$ and $R_{\max }$, to describe failure behaviors, by completely turning off the stiffening effect from the switching function. The choice of cutoffs can successfully describe complex carbon network including pristine graphene without stiffening near the failure point [43]. Fig. S11 A and B show the monolayer and bilayer graphene models used for the MD simulations. The size of each graphene model is $40 \times 40$ 
$\mathrm{nm}^{2}$. The bilayer graphene is of AB-stacking, and the interlayer distance was set to $3.4 \AA$. By setting the atomic resolution to describe the bond breaking of graphene and vdW interactions between the layers as invariable, we implicitly handle the mass transport of mixed liquid due to Maragoni effect on the liquid surface as a part of spherical droplet exposed on the flat liquid surface by utilizing a moving spherical indenter. We approximate the hardness of the droplet using a simple equation for a spherical particle on the liquid surface [44] in Fig. S11C. From the small deformation, $d$, on the liquid surface due to the spherical solid, we obtain a linear relation from the vertical component of the surface tension on a spherical particle, $F=2 \pi R \gamma \sin ^{2} \theta$, by applying the approximation, $\sin ^{2} \theta \sim \frac{2 d}{R}$

$$
F=4 \pi \gamma d
$$

where the $\gamma$ is surface tension of liquid. The surface tension of water is typically 72 dynes $/ \mathrm{cm}(\sim 0.0078 \mathrm{eV} / \AA)$ at $293 \mathrm{~K}[45]$. In simulations, we set the linear spring for spherical indenter, $k$ to $\sim 0.005 \mathrm{eV} / \AA$, as the mixture with alcohol reduces the surface tension.

We further assume that the graphene is initially contacted with the relatively flat liquid surface. The liquid surface is also implicitly described by Lennard Jones (LJ) wall as

$$
E=\varepsilon\left[\frac{2}{15}\left(\frac{\sigma}{r}\right)^{9}-\left(\frac{\sigma}{r}\right)^{3}\right]
$$

where the LJ parameters are obtained from previous graphene and water interface study [46] by using the mixing rule as $\varepsilon=0.06 \mathrm{eV}$, and $\sigma=3.2 \AA$ with $r_{\text {cut }}=3 \sigma$. We restricted the movement of boundaries in the $z$-direction. After we relaxed the systems with NVT ensemble at $300 \mathrm{~K}$ for 20 ps using energy minimization principle, a sphere indenter with $R=5 \mathrm{~nm}$ moves with a speed $0.2 \AA / \mathrm{ps}(20 \mathrm{~m} / \mathrm{s})$ along the $y$ direction, as shown in Fig. S11D. The center of the sphere is located under the water surface with a $10 \mathrm{~nm}$ distance. 


\section{REFERENCES AND NOTES}

\section{References}

1. Ohta, T., et al., Controlling the electronic structure of bilayer graphene. Science, 2006. 313(5789): p. 951-954.

2. Mayorov, A., et al., Interaction-driven spectrum reconstruction in bilayer graphene. Science, 2011. 333(6044): p. 860-863.

3. Gao, Y., et al., Ultrahard carbon film from epitaxial two-layer graphene. Nature nanotechnology, 2018. 13(2): p. 133.

4. Yankowitz, M., et al., Tuning superconductivity in twisted bilayer graphene. Science, 2019. 363(6431): p. 1059-1064.

5. Cao, Y., et al., Unconventional superconductivity in magic-angle graphene superlattices. Nature, 2018. 556(7699): p. 43.

6. Codecido, E., et al., Correlated insulating and superconducting states in twisted bilayer graphene below the magic angle. 2019. 5(9): p. eaaw9770.

7. Sharpe, A.L., et al., Emergent ferromagnetism near three-quarters filling in twisted bilayer graphene. 2019. 365(6453): p. 605-608.

8. Zhang, Y., et al., Direct observation of a widely tunable bandgap in bilayer graphene. Nature, 2009. 459(7248): p. 820.

9. Tang, T.-T., et al., A tunable phonon-exciton Fano system in bilayer graphene. Nature nanotechnology, 2010.5(1): p. 32.

10. Zhou, S.Y., et al., Substrate-induced bandgap opening in epitaxial graphene. Nature materials, 2007. 6(10): p. 770.

11. Zhang, J. and J. Zhao, Mechanical properties of bilayer graphene with twist and grain boundaries. Journal of Applied Physics, 2013. 113(4): p. 043514.

12. Liu, L., et al., High-yield chemical vapor deposition growth of high-quality large-area AB-stacked bilayer graphene. ACS nano, 2012. 6(9): p. 8241-8249.

13. Liao, L., et al., van Hove singularity enhanced photochemical reactivity of twisted bilayer graphene. Nano letters, 2015. 15(8): p. 5585-5589.

14. Yin, J., et al., Selectively enhanced photocurrent generation in twisted bilayer graphene with van Hove singularity. Nature communications, 2016. 7(1): p. 1-8.

15. Ma, W., et al., Interlayer epitaxy of wafer-scale high-quality uniform AB-stacked bilayer graphene films on liquid Pt 3 Si/solid Pt. Nature Communications, 2019. 10(1): p. 2809.

16. Reina, A., et al., Growth of large-area single-and bi-layer graphene by controlled carbon precipitation on polycrystalline Ni surfaces. Nano Research, 2009. 2(6): p. 509-516.

17. Wu, Y., et al., Growth mechanism and controlled synthesis of AB-stacked bilayer graphene on $\mathrm{Cu}-\mathrm{Ni}$ alloy foils. ACS Nano, 2012. 6(9): p. 7731-7738.

18. Huang, M., et al., Large-area single-crystal AB-bilayer and ABA-trilayer graphene grown on a $\mathrm{Cu} / \mathrm{Ni}$ (111) foil. Nature nanotechnology, 2020. 15(4): p. 289-295.

19. Hao, Y., et al., Oxygen-activated growth and bandgap tunability of large single-crystal bilayer graphene. Nature Nanotechnology, 2016. 11(5): p. 426.

20. Humphreys, C., Controlling crystal growth. Nature, 1989. 341(6244): p. 689-689.

21. Gurioli, M., et al., Droplet epitaxy of semiconductor nanostructures for quantum photonic devices. Nature materials, 2019: p. 1.

22. Fang, W., et al., Asymmetric growth of bilayer graphene on copper enclosures using low-pressure chemical vapor deposition. 2014. 8(6): p. 6491-6499.

23. Hao, Y., et al., The role of surface oxygen in the growth of large single-crystal graphene on copper. Science, 2013. 342(6159): p. 720-723.

24. Ta, H.Q., et al., Stranski-Krastanov and Volmer-Weber CVD growth regimes to control the stacking order in bilayer graphene. Nano letters, 2016. 16(10): p. 6403-6410. 
25. Yoon, T., et al., Direct measurement of adhesion energy of monolayer graphene as-grown on copper and its application to renewable transfer process. Nano Letters, 2012. 12(3): p. 14481452.

26. Li, S., et al., The evolving quality of frictional contact with graphene. Nature, 2016. 539(7630): $\mathrm{p}$. 541-545.

27. Crank, J., The mathematics of diffusion. 1979: Oxford university press.

28. Zhou, H., et al., Chemical vapour deposition growth of large single crystals of monolayer and bilayer graphene. Nature Communications, 2013. 4: p. 2096.

29. Kim, K., et al., Raman spectroscopy study of rotated double-layer graphene: misorientation-angle dependence of electronic structure. Physical review letters, 2012. 108(24): p. 246103.

30. Papageorgiou, D.G., I.A. Kinloch, and R. Young, Mechanical properties of graphene and graphene-based nanocomposites. Progress in Materials Science, 2017. 90: p. 75-127.

31. Leong, W.S., et al., Paraffin-enabled graphene transfer. Nature Communications, 2019. 10(1): p. 867.

32. Wang, H., et al., Low-temperature copper bonding strategy with graphene interlayer. ACS nano, 2018. 12(3): p. 2395-2402.

33. MacKay, G. and S. Mason, The Marangoni Effect and Liquid/Liquid Coalescence. Nature, 1961. 191(4787): p. 488.

34. Pasternak, I., et al., Graphene films transfer using marker-frame method. Aip Advances, 2014. 4(9): p. 097133.

35. Kresse, G. and J. Hafner, Ab initio molecular dynamics for liquid metals. Physical Review B, 1993. 47(1): p. 558.

36. Kresse, G. and J. Hafner, Ab initio molecular-dynamics simulation of the liquid-metalamorphous-semiconductor transition in germanium. Physical Review B, 1994. 49(20): p. 14251.

37. Blöchl, P.E., Projector augmented-wave method. Physical review B, 1994. 50(24): p. 17953.

38. Perdew, J.P., M. Ernzerhof, and K. Burke, Rationale for mixing exact exchange with density functional approximations. The Journal of chemical physics, 1996. 105(22): p. 9982-9985.

39. Thonhauser, T., et al., Van der Waals density functional: Self-consistent potential and the nature of the van der Waals bond. Physical Review B, 2007. 76(12): p. 125112.

40. Plimpton, S., Fast parallel algorithms for short-range molecular dynamics. Journal of computational physics, 1995. 117(1): p. 1-19.

41. Brenner, D.W., et al., A second-generation reactive empirical bond order (REBO) potential energy expression for hydrocarbons. 2002. 14(4): p. 783.

42. Stuart, S.J., A.B. Tutein, and J.A. Harrison, A reactive potential for hydrocarbons with intermolecular interactions. The Journal of chemical physics, 2000. 112(14): p. 6472-6486.

43. Jung, G.S. and M.J. Buehler, Multiscale mechanics of triply periodic minimal surfaces of threedimensional graphene foams. Nano Letters, 2018. 18(8): p. 4845-4853.

44. Rapacchietta, A. and A. Neumann, Force and free-energy analyses of small particles at fluid interfaces: II. Spheres. Journal of Colloid Interface Science, 1977. 59(3): p. 555-567.

45. Vargaftik, N., B. Volkov, and L. Voljak, International tables of the surface tension of water. Journal of Physical Chemical Reference Data, 1983. 12(3): p. 817-820.

46. Zhou, H., et al., Understanding controls on interfacial wetting at epitaxial graphene: Experiment and theory. Physical Review B, 2012. 85(3): p. 035406. 


\section{ACKNOWLEDGEMENTS}

The authors acknowledge Y. Zhang for the help with Transmission Electron Microscope. The authors acknowledge W. S. Leong for the help with Raman measurements and manuscript editing.

Author contributions: H. W., J. K. conceived the overall project. H.W., M.H., T.P., and J.K. conceived, performed, and analyzed the bilayer graphene growth and transfer. Z.Y. and A.A.-G. performed, and analyzed the firstprinciple calculations. G.S.J. and M.J.B. performed and analyzed the MD simulations. Q.S. and G.C. performed the artificial-intelligence-assisted Raman data analysis. H.W., Z.Y., G.S.J., Q.S. and J.K. wrote the manuscript. All authors contributed to the overall scientific interpretation and edited the manuscript.

Funding: The work is partially performed at MIT Microsystems Technology Laboratories (MTL), MIT Materials Research Laboratories (MRL) and Center for Nanoscale Systems (CNS), Harvard University. H.W. and J.K. acknowledges the support from the Center for Energy Efficient Electronics Science (NSF Award No. 0939514), J.K. Acknowledges the support from the AFOSR FATE MURI, Grant No. FA9550-15-1-0514. Z.Y. and A.A.-G. acknowledge the support from the Nanoporous Materials Genome Center by the U.S. Department of Energy, Office of Science, Office of Basic Energy Sciences under award number DE-SC0008688. Q.S. and G.C. acknowledge the support from Office of Naval Research under MURI grant N00014-16-1-2436. G.S.J. and M.J.B. also acknowledge support from AFOSR FATE MURI, Grant No. FA9550-15-1-0514 and the US Department of Defense, Office of Naval Research (N00014-16-1-233). The authors acknowledge the assistance of data collection and analysis from Dr. Wei Sun Leong and Dr. Yong Zhang.

Competing interests: The authors declare no competing interests. 

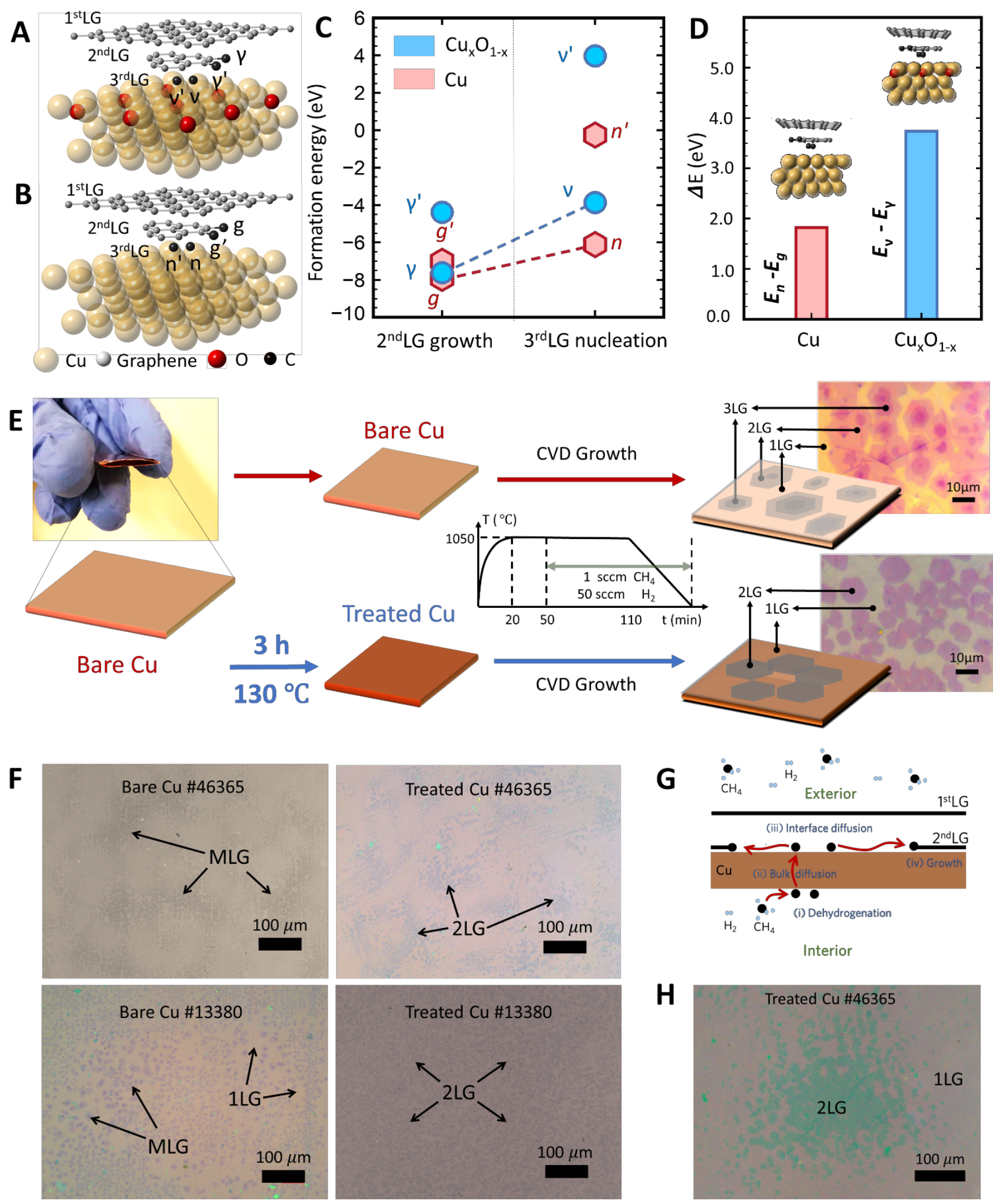

Fig. 1. Our Frank-van der Merwe (FM) mode-enabled bilayer graphene (2LG) growth. (A and B) Atomic geometries of the 2LG growth behavior on (A) pure and (B) modified $\mathrm{Cu}$ substrates. (C) Formation energies of the $2^{\text {nd }}$ layer graphene $\left(2^{\text {nd }} L G\right)$ growth and the $3^{\text {rd }}$ layer graphene $\left(3^{\text {rd }} L G\right)$ nucleation, on pure and modified $\mathrm{Cu}$, respectively. (D) Energy difference, $\Delta \mathrm{E}$, correspond to suppression of the $3^{\text {rd }} \mathrm{LG}$ nucleation for both pure and modified $\mathrm{Cu}$. (E) Schematics showing the chemical vapor deposition (CVD) of 2LG on bare and treated $\mathrm{Cu}$. (F) 
Optical images of 2LG samples transferred on separate $\mathrm{SiO}_{2} / \mathrm{Si}$ substrates, grown using different types of $\mathrm{Cu}$ foils as indicated. 1LG, 2LG and multilayer graphene (MLG) regions are labeled. These samples were grown for $1 \mathrm{~h}$. (G) Schematic illustrating the 2LG growth mechanism in the CVD Cu "pocket" approach. (H) Typical optical image of a $2 \mathrm{LG}$ sample transferred on $\mathrm{SiO}_{2} / \mathrm{Si}$ substrate, grown for $3.33 \mathrm{~h}$ with the treated $\mathrm{Cu}$, showing galaxylike morphologies of $2 \mathrm{LG}$. 

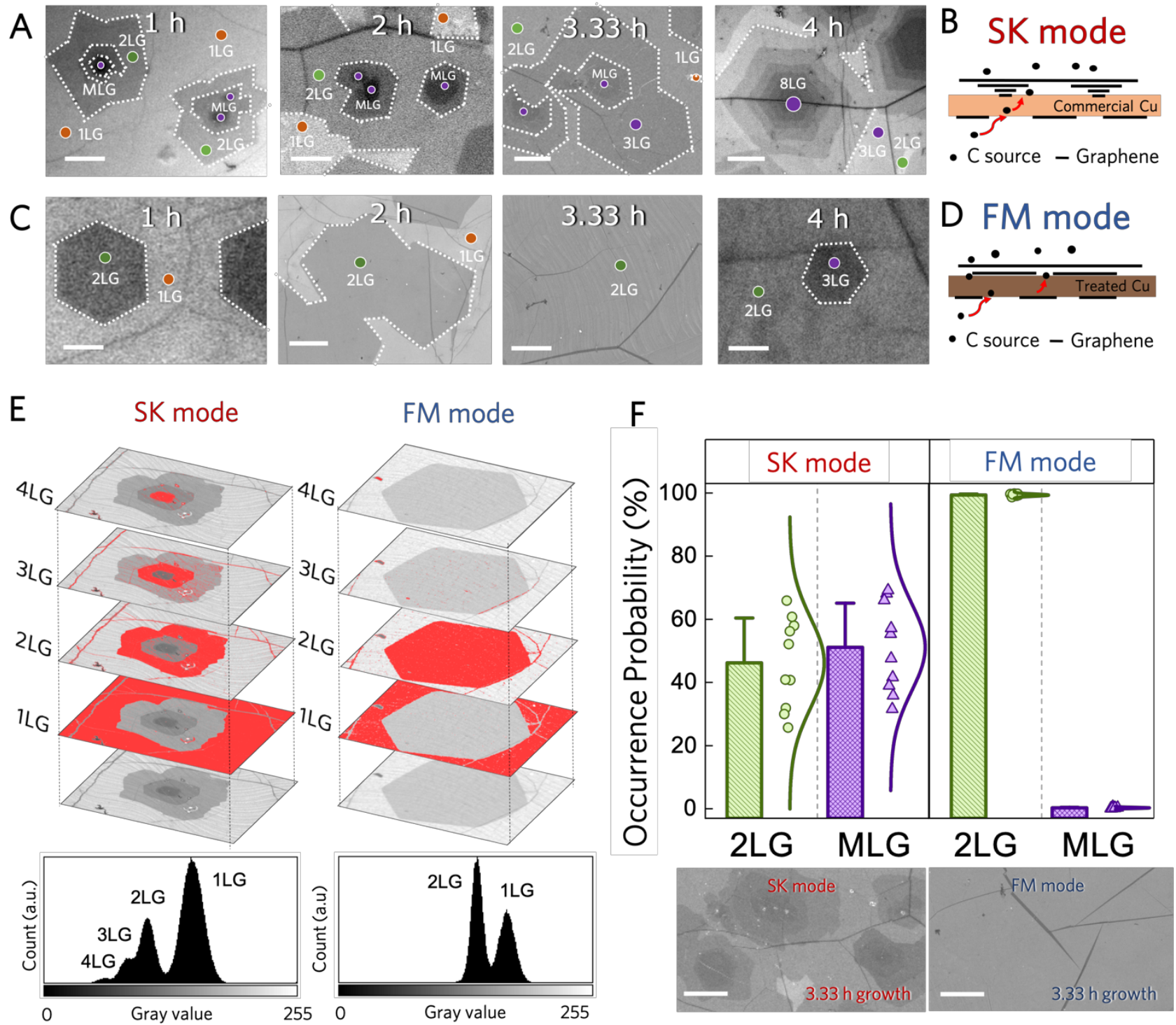

Fig. 2. Comparison of SK- and FM-mode grown 2LG films. (A and C) Microscopic images of graphene synthesized on (A) commercial $\mathrm{Cu}$ and $(\mathbf{C})$ pre-treated $\mathrm{Cu}$ (cut from the same piece of commercial $\mathrm{Cu}$ foil in (A)), respectively, for different growth durations as indicated (i.e. $1 \mathrm{~h}, 2 \mathrm{~h}, 3.33 \mathrm{~h}$, and $4 \mathrm{~h}$ ). Samples shown in these optical images are graphene transferred on $\mathrm{Si}_{/} \mathrm{SiO}_{2}$. (B and $\left.\mathbf{D}\right)$, Schematics illustrating the cross-sectional views of 2LG growth behaviors in Stranski-Krastanov (SK) and Frank-van der Merwe (FM) growth modes, on commercial and treated $\mathrm{Cu}$, respectively. (E) Bottom, pixel counts as a function of gray values, for a microscopy image of typical SK- and FM-mode grown graphene film, respectively. Top, statistical labelling for each layer of graphene. Both samples were grown for 1 h. (F) Bottom, images of a typical graphene grown with $3.33 \mathrm{~h}$, for both SK and FM modes. Top, occurrence probability for different graphene layers that was statistically identified using the method shown in E, with 20 typical images, with sizes of at most $40 \times 40 \mu \mathrm{m}^{2}$, taken from both SK- and FM-mode grown samples (10 each). Multilayer graphene (MLG) represents 3LG, 4LG, and above. The occurrence probability of 1LG in these samples are not shown, they are $2.64 \%$ and $0.29 \%$ for SK mode and FM mode respectively. All scale bars are 5 $\mu \mathrm{m}$. 

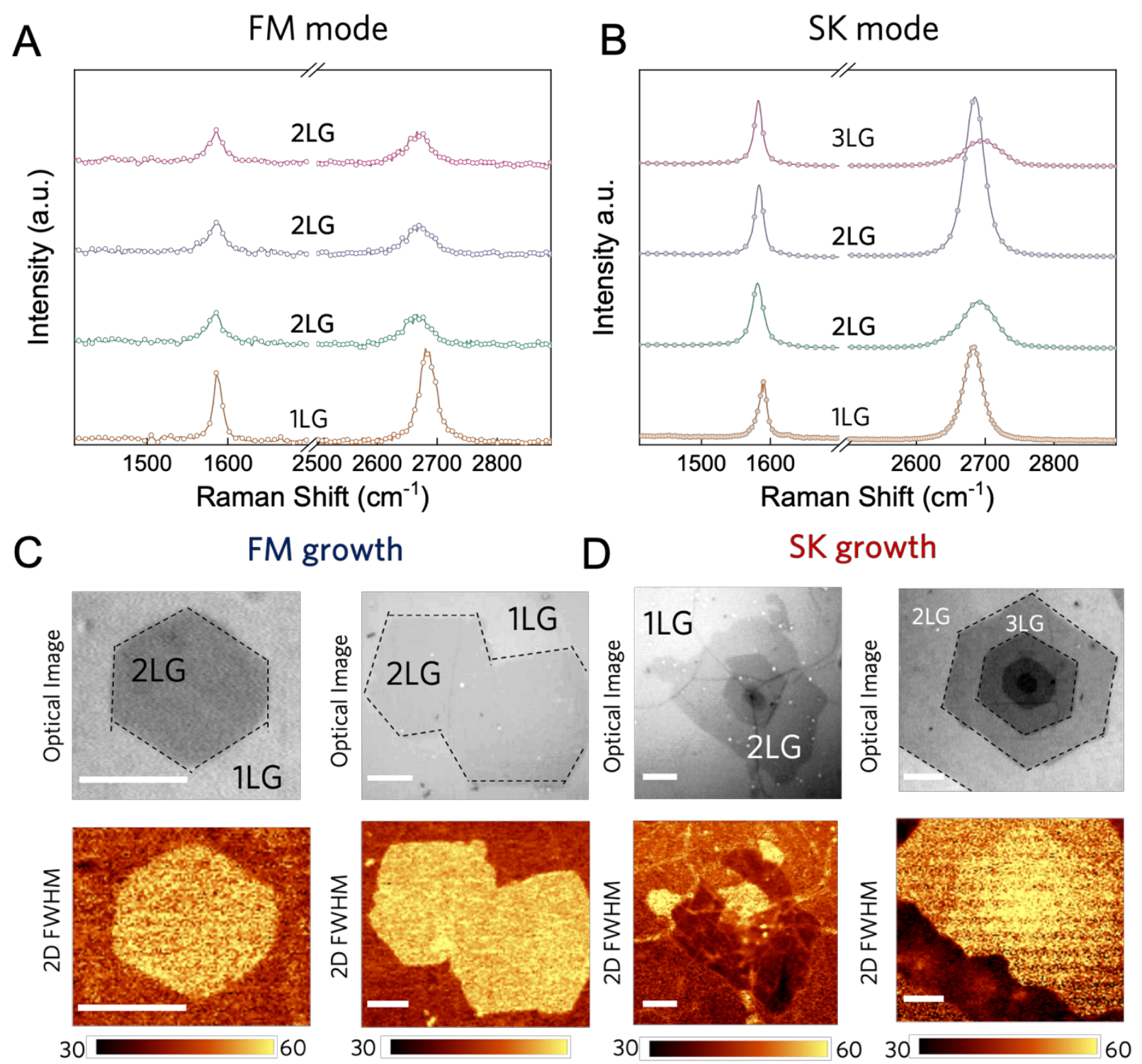

Fig. 3. Raman analysis of graphene samples grown via FM and SK mode. (A) Raman spectra taken at different positions on a typical FM-mode grown 2LG sample (B) Raman spectra taken at different positions on a typical SKmode grown 2LG sample (C) Microscopic images of typical FM-mode grown sample and the corresponding Raman map of 2D band's full width at half maximum (2D FWHM). (D) Microscopic images of typical SK-mode grown sample and the corresponding Raman map of 2D FWHM. All scale bars are $5 \mu \mathrm{m}$. 


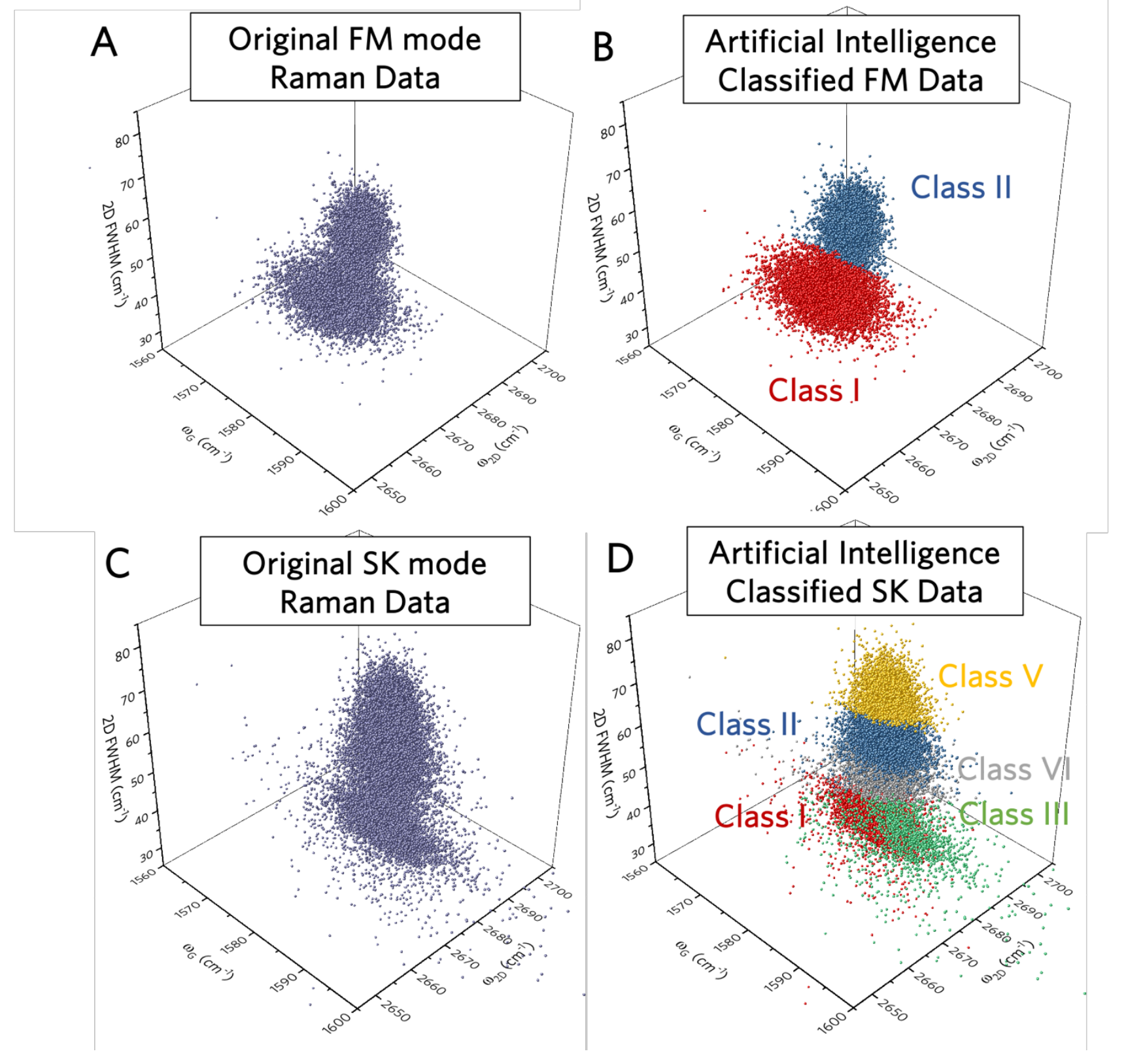

Fig. 4. Artificial-Intelligence-assisted Raman analysis. (A) 3D plot of the $\mathrm{G}$ band position $\left(\omega_{G}\right), 2 \mathrm{D}$ band position $\left(\omega_{2 D}\right)$, and 2D FWHM, extracted from 18,000 Raman spectra of the FM-mode grown sample. (B) 3D plot showing artificial-intelligence-classified data: Class I (red) and II (blue), which can be identified as the Raman data of 1LG and 2LG, respectively. (C) 3D plot of the $\omega_{G}, \omega_{2 D}$, and 2D FWHM, extracted from 20,250 Raman spectra of the SK-mode grown sample. (D) 3D plot showing artificial-intelligence-classified data: Class I (red), II (blue), III (light green), IV (grey), and V (yellow), which can be identified as the Raman data of 1LG, quasi-AB-stacked 2LG, larger-twisting-angle 2LG, 3LG, and MLG respectively. 
A

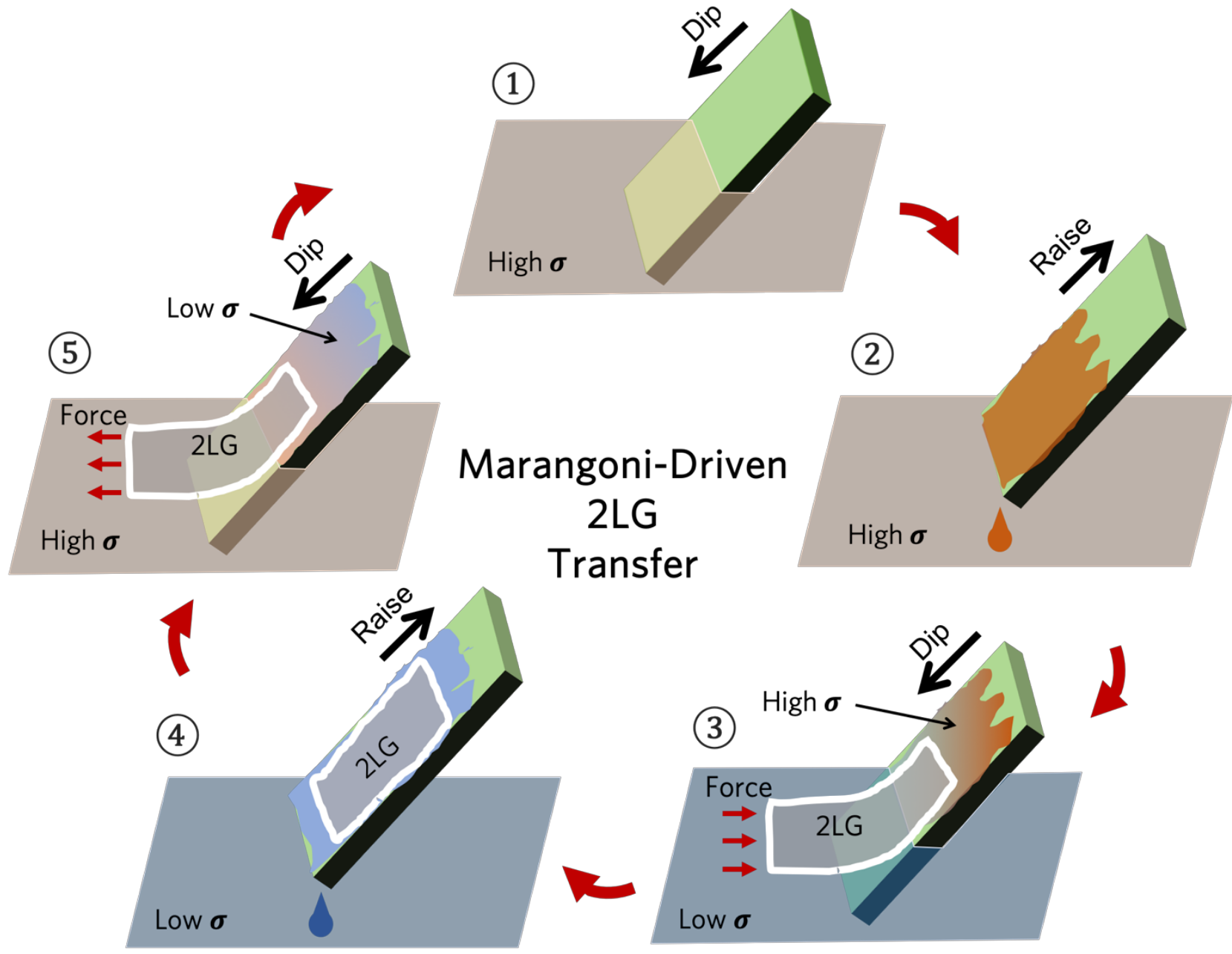

B

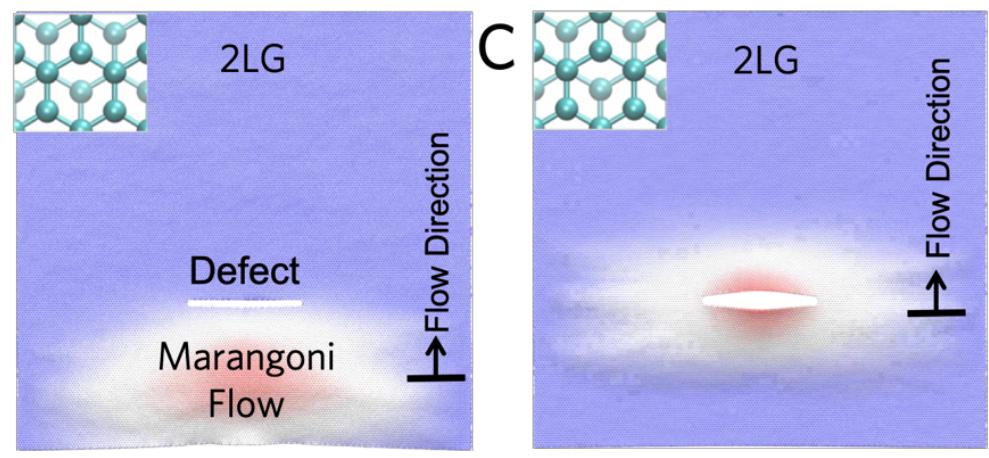

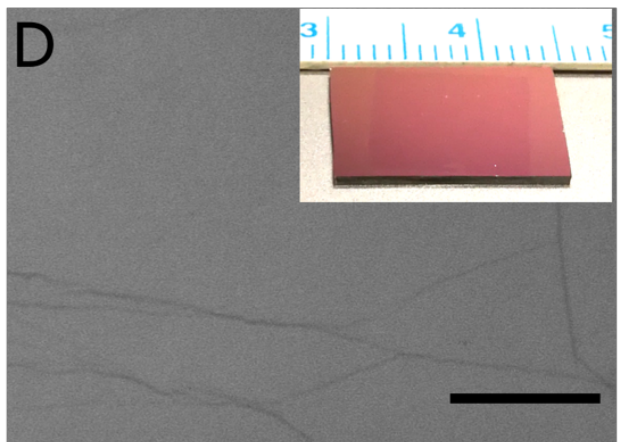

Fig. 5. Support-free $2 \mathrm{LG}$ graphene transfer driven by Marangoni effect. (A) Schematics showing the concept of our Marangoni-driven graphene transfer process. A graphene sample was first floated on a liquid (either $\mathrm{Cu}$ etchant or DI water). A flat scoop (e.g. wafer or glass slide) was wetted with a liquid of higher surface tension (high- $\boldsymbol{\sigma}$ ) and immediately dip into the container with graphene sample. Driven by Marangoni flow, the graphene will automatically climb to the flat scoop. Once the flat scoop was brought back to the high- $\boldsymbol{\sigma}$ liquid, the graphene sample gradually glides from the flat scoop and stays on the liquid surface. (B to C) Snapshots of a simulated Marangoni flow passing through a 2LG film (see Movie S2). The 2LG remains robust under the Marangoni flow, even in the presence of atomic defects. Insets: Atomic structures of an AB-stacked 2LG used in the molecular 
dynamic simulation. (D) SEM image of a typical 2LG sample transferred on a $\mathrm{Si} / \mathrm{SiO}_{2}$ substrate by our supportfree Marangoni-driven approach. Inserted figure: optical image demonstrating centimeter-scale transfer capability of the support-free Marangoni-driven transfer. Scale bar is $20 \mu \mathrm{m}$. 